\title{
CLOSE ENCOUNTERS OF THE STRESSFUL KIND: AFFECTIVE, PHYSIOLOGICAL, AND BEHAVIORAL REACTIONS TO THE EXPERIENCE OF CROWDING
}

Tedra A. Walden

Donelson R. Forsyth

ABSTRACT: Ninety-six adults participated in a study of the joint effects of interpersonal density and an additional stressor. Experiential reports, psychophysiological indices, and task performance were assessed. Subjects seated either close together or far apart worked on a series of tasks while either expecting a stressful event or not expecting the stressful event. Perceptions of crowding were influenced only by interpersonal distance; those seated close together reported feeling more crowded than those seated far apart. However, if subjects were both crowded and expected a stressful event they reported feeling more stressed and evidenced psychophysiological signs of anxiety. Relevance of these results for an additive stress model is discussed but the model is rejected and an interactional model of crowding and stress effects is proposed. Evidence for processes of social comparison fear reduction was found in some crowded subjects.

One major stumbling block to the development of a systematic theory of crowding has been the failure to satisfactorily define what is meant by "crowding." Early researchers defined crowding in terms of physical density, the number of individuals per unit of space (Calhoun, 1962; Christian, 1961; Freedman, Heshka, \& Levy, 1975; Freedman, Klevansky, \& Ehrlich, 1971; Calle, Gove, \& McPherson, 1972; Griffitt \& Veitch, 1971). Subsequent investigations have failed to find negative effects to be reliably associated with density and, as the definition of density raises a number of conceptual problems (Loo, 1973), alternate definitions have been sought.

Tedra A. Walden is affiliated with the Department of Psychology and Human Development, Peabody Box 154, Vanderbilt University, Nashville, TN 37203. Reprint requests should be addressed to her there. Donelson $R$. Forsyth is at Virginia Commonwealth University. 
Stokols (1972) has argued that there are important differences between density, a physical parameter, and the experience of crowding, a psychological state. He defined crowding as an experiential state "in which the restrictive aspects of limited space are perceived by the individuals exposed to them" (p. 275, italics added). Stokols proposed that the subjective feeling of crowding is the proper independent variable of interest in spatial research.

Following Stokols' suggestion, investigators have recently favored the use of self-report assessments of subjective crowding, of ten responses to an item or scale asking, "How crowded do you feel?" The subjective feeling of crowding is commonly viewed as a manipulation check of density conditions (e.g., Epstein \& Karlin, 1975; Evans, 1979; Schopler, McCallum \& Rusbult, Note 1). That is, the self-reported feeling of crowding is conceptualized as validation that sufficiently dense conditions have been imposed. Without the reported feeling of crowding, no effects of "crowding" can be said to occur. Few have explicitly considered that the feeling of crowding is but one possible effect of high density and that other noncognitive responses may occur independently of the reported feeling of crowding. However, Worchel and Teddlie (1976) and Worchel and Yohai (1979) did suggest that the crowding attribution may be blocked by relatively minor external variations. They emphasized that perceived crowding is a process of cognitive labeling that depends upon an individual experiencing a state of arousal and attributing that arousal to the uncomfortably close presence of others. There is little evidence to suggest that the subjective feeling of crowding necessarily parallels other effects of high density or that effects of density may not occur in the absence of the attribution.

Nisbett and Wilson (1977) have argued that people are often unable to assess whether a given stimulus has influenced their behavior at all, and, if so, what that effect has been. Nisbett and Wilson propose that when people are asked to report on the influence of a particular stimulus on a response they do so, not by assessing internal mediating responses, but by applying causal theories about the effects of that type of stimulus on that type of response. It is thus important to consider whether self-reports of crowding are accurate reflections of a person's "total" responses to high density or are simply a cognitive appraisal of objective density conditions.

Research indicates that high density is associated with a variety of effects including subjective experience, physiological responses, and task performance. Dense conditions do often 
produce subjective, self-reported feelings of crowding (Epstein \& Karlin, 1975; Griffitt \& Veitch, 1971; Langer \& Saegert, 1977; Sundstrom, 1975; Worchel \& Teddlie, 1976), although in some high density conditions subjects do not feel crowded (Worchel \& Teddlie, 1976). Crowded conditions have been shown to be associated with such physiological effects as decreased skin resistance, increased respiratory rate, elevated cortisol levels, higher blood pressure and increased heart rate (Aiello, Epstein, \& Karlin, 1975; D'Atri, 1975; Evans, 1979; McBride, King, \& James, 1965; Sequin, 1967; Epstein \& Aiello, Note 2; Heshka \& Pylpuk, Note 3). Some studies, however, have found no significant changes in psychophysiological indices with high density (Aiello et al., 1975; Dabbs, 1971; Efran \& Cheyne, 1974; McCallum, Rusbult, Hong, Walden, \& Schopler, 1979). A third response that may be influenced by crowding is task performance. Decrements in task performance have generally been found when the tasks assessed were complex (Aiello, et al., 1975; Evans \& Howard, 1973; Evans, 1979; Barefoot \& Kleck, Note 4) but not if simple tasks were used (Epstein \& Karlin, 1975'; Evans \& Howard, 1973; Barefoot \& Kleck, Note 4). Still other work shows little evidence of decrements on either simple or complex tasks (Freedman et al., 1971; Sherrod, 1974).

Few studies of crowding have included direct measures of subjective cognitive responses, physiological effects, and task performance (c.f. Aiello et al., 1975; Aiello, Nicosia, \& Thompson, 1979). Many studies assess effects on one or two types of response, yet generalize conclusions beyond the type of response assessed. Only indirect evidence may be used to assess one or more types of response. For instance, Worchel and Teddlie (1976) used data such as whether a leader emerged in the group and distortions in time perceptions to indicate subjects' state of arousal. It is uncertain whether interpersonal density affects all three response systemscognitive, physiological, and task-similarly or has differential effects on the three. One purpose of the present study was to assess the effects of high interpersonal density on all three domains of functioning.

A second relatively unexplored issue in the study of crowding concerns the effects of crowding on persons who are exposed to a second source of stress or arousal concurrently with excessive

'However, Evans (1979) has criticized Epstein and Karlin's task performance measures as being possibly inappropriate because all tasks were assessed after exposure to crowded conditions and not concurrently with crowding. 
interpersonal proximity. Griffitt and Veitch (1971) combined two sources of stress, high temperature and high density, ${ }^{2}$ and found main effects for each stressor separately but no interactions between the two stressors, suggesting that the effects of the two stressors combined additively. Worchel and Yohai (1979) studied joint effects of crowding and noise. A more general test of the combined effects of density and another stressor might be provided if a general, nonspecific source of stress could be produced independently of crowding.

We sought to produce a non-specific state of arousal or stress by giving some subjects reason to expect that they would experience from a source unrelated to crowding some of the same stressful autonomic effects that have been purported to result from crowding: increased heart rate, increased respiration, and other anxiety-like symptoms. Combining the presence or absence of the two sources of stress factorially, we attempted to test the separate and combined effects of high density and an additional general state of stress or arousal. Furthermore, we wanted to make this source of stress "attributionally salient" to subjects. Worchel and Teddlie (1976) suggested that subjective crowding could be reduced if subjects were distracted from focusing on the close presence of others. They used a "pleasant" distraction (art work) and concluded that both subjective crowding and stress were reduced with distracted. We sought to extend this work by using a "negative" distraction to investigate: 1) whether subjects would consider themselves to be less subjectively crowded when distracted from crowding (e.g., led to misattribute the symptoms that they might experience from high density to another possible cause) and 2) whether the two sources of stress combined additively, producing independent negative effects. Finally, we assessed the effects of the two stressors on the three separate types of response-cognitions, task performance, and psychophysiological measures.

\section{METHOD}

\section{Subjects}

Forty-eight male and 48 female introductory psychology students participated in same-sex groups of six. Two female experimenters

\footnotetext{
${ }^{2} \mathrm{Griffitt}$ and Veitch used changes in group size to manipulate density, confounding the effects of increased number of persons and density.
} 
working together conducted all the sessions. All groups were composed of unacquainted individuals.

\section{Procedure}

The experiment, entitled "Medical Research Experiment 23-C," was conducted in a large teaching hospital several blocks from the Psychology Department. Participants believed that although the study was not being conducted under the auspices of the Psychology Department, their participation would be transferred to that department for partial fulfillment of the laboratory research option. Upon arrival, subjects were seated by the experimenters in an anteroom containing six comfortable armchairs and several tables of medical equipment. Subjects were requested not to smoke or to talk during this period. The experimenters, dressed in traditional nursing attire, waited five minutes after the arrival of the sixth subject before starting the research procedure.

Before relating the supposed purposes of the experiment to the participants, the experimenters identified themselves as nurses and distributed letter (A through F) identification badges. Then, while the subjects remained seated, one nurse recorded each subject's pulse, respiration and blood pressure. The nurses explained that the project was designed to gather information about the cognitive and physiological effects of vitamin compounds. Certain supplements that had been developed by the staff at the hospital and used with humans for several years had been found to produce measurable effects after a short period of time. The current study would focus on changes in physiological states, perceptual abilities, and intellectual aptitudes. Therefore, each person would be asked to take a small dose of the vitamin and then have his or her reactions recorded. None of the subjects refused to participate.

Before taking the "medication" all subjects were asked to sign an approval form stating that they had agreed to take the drug. The drug was described to all subjects as "harmless and quite mild." Those subjects given side effects information, however, were told by the experimenters that "one of the properties of the drug is that it acts as a mild stimulant to your nervous system and therefore quite a few of our subjects experience mild effects. These side effects are transitory; they last only for about 30 minutes. What you may experience is a slightly accelerated heartrate and breathing; you may feel warm, flushed, and shaky. In no case have these symptoms been severe, but they are reported fairly regularly among our patients." Subjects given no side effects information were simply told, "It has been shown to have no side effects whatever. Therefore, you should not even be aware that the drug is in your system." The appropriate side effects information was reiterated in the approval form signed by each subject. 
After the consent forms were collected, $20 \mathrm{cc}$. of the vitamin supplement were administered to each subject in a plastic medicine cup. The "drug," actually a weak mixture of orange-flavored gelatin dissolved in water, was taken in measured dosages from a standard $400 \mathrm{cc}$. medicine bottle. After ingesting their doses, subjects were seated by the second experimenter in an adjoining room (approximately $8 \times 10$ meters). In the center of the room was a circle of armless chairs and a microphone was suspended from the ceiling. In the far interpersonal distance condition the chairs were placed 40 centimeters apart (as measured from the inner corners of adjacent chairs), while in the close interpersonal distance condition the corners of adjacent chairs touched. ${ }^{3}$ In the close condition, one experimenter pulled a chair from the circle to allow subjects to be seated inside the circle, pushing it under the last person to be seated. Subjects were told not to move the chairs because, as evidenced by the microphone, parts of the session would be recorded (see Worchel \& Teddlie, 1976).

While seated in the circle, subjects completed a medical history form that requested a variety of information about past diseases, illnesses, and general physical health. Then, subjects were told that enough time had elapsed for the drug to have entered their systems and that measurements could now be taken. A series of group and individual tasks was structured such that the experimenter completely controlled the interactants' behaviors but also obtained measures of task performance. The tasks included a group measure of recall and individual measures of anagram solving ability, recall, creativity, and punitiveness. When the tasks were finished subjects completed a questionnaire requesting information about their symptoms, the amount of stress they had experienced, and their perceptions of the seating arrangements. Physiological measures were again taken, after which subjects were debriefed, thanked, and cautioned not to discuss the experiment with anyone.

\section{Dependent Measures}

Physiological indices. Systolic and diastolic blood pressure were measured using the standard procedure (Lywood, 1967) with a sphygmomanometer, stethoscope, and an occular cuff around the right arm. Pulse was measured at the wrist by listening for 15 seconds with a stethoscope, and respiration was counted unobtrusively for 15 seconds following the pulse.

Self-reported symptoms. Subjects were asked to indicate if they experienced any effects of the medication they had taken by indicating on

${ }^{3}$ No physical contact was present in either condition. 
a 7-point scale the extent to which they had experienced each of 13 symptoms. Nine of the symptoms were relevant to the effects described to the subjects in the side effects information conditions and four were filler items.

Perceived crowding and stress. The four items used by Worchel and Teddlie (1976) to assess the experience of crowding were included on the questionnaire (How comfortable did you feel during the research procedure?, How confined. . . ? How ill at ease ... ? and How crowded. . . ?). In addition, subjects rated how stressed they felt, how much they liked the other group members and the group experience, and the friendliness, nervousness, and aggressiveness of the group members. All items were measured on 12-point scales with five labeled gradations ranging from "not all" to "extremely." Also, subjects rated the experimental setting on six 7-point bi-polar adjective scales and rated themselves on 15 7-point bi-polar adjective scales.

Task performance. Problem solving, memory, creativity, and punitiveness were assessed. The anagrams task consisted of 15 scrambled words rated by Feather (1967) at approximately $65 \%$ difficulty $(65 \%$ of the people tested could solve each one). A subject's score was computed by adding the number of anagrams correctly solved within the two minutes allotted.

One group and one individual memory task were used. In both, a list of 30 words was read aloud at a rate of one word every two seconds by an experimenter. In the individual task each group member tried to individually recall and write down all the words from the list. On the group task, members took turns around the circle, volunteering words until no words could be recalled. The creativity task involved creating different uses for an old wooden ladder, a metal coat hanger, an automobile tire, and a coconut. The total number of different uses each subject listed (added across the four items) measured creativity. Finally, each person read the case history of a young juvenile delinquent, Johnny Rocco (Worchel \& Teddlie, 1976). Subjects read the narrative individually and indicated on the accompanying scale which of seven alternative treatment programs they would recommend. The alternatives varied along a punitiveness dimension from benign or loving to totally punitive.

Causal attributions. Subjects rated on 12-point scales the extent to which their task performance was influenced by the medication, the presence of the other people and distractions. Subjects also rated the extent to which the feelings they experienced were due to the medication, the presence of others, the fact of participating in an experiment or "other" factors. 


\section{RESULTS}

\section{Interpersonal Distance and the Perception of Crowding ${ }^{4}$}

It was hypothesized that in the current experimental situation close physical proximity would cause subjects to perceive themselves to be more crowded. Two (interpersonal distance: close vs. far) $X$ two (information: side effects vs. no side effects) $X$ two (sex) multivariate and univariate analyses of variance confirmed this prediction, replicating previous findings (Epstein \& Karlin, 1975; Worchel \& Teddlie, 1976). Subjects' perceptions of crowding were influenced only by interpersonal proximity, with subjects seated closer together rating themselves on the four crowding items as more crowded than subjects seated farther apart, $F(4,85)$ $=15.70, p<.05$. Univariate analyses indicated that close subjects, in comparison to those seated far apart, rated themselves as more crowded during the research and more confined (see Table 1 for $F$-ratios and means). In addition, only a main effect of distance was obtained multivariately on subjects' responses to the six bi-polar adjectives pertaining to perceptions of the experimental setting, $F(6,83)=6.90, p<.05$. Subjects seated close together, in comparison to those seated far apart, rated the setting as more uncomfortable, more confining, more restrictive, more poorly arranged, and more crowded (see Table 1).

\section{Situational Information and Stress}

Analyses of both subjective and objective measures of stress yielded support for the prediction that under crowded conditions subjects would be more stressed if information relevant to the interpretation of the experienced psychological state was negative (noxious) rather than neutral (innocuous). A marginally significant interaction of distance and information was obtained on the item "How stressed did you feel during the research procedure?" The means for this interaction, presented in Table 2, show that stress

\footnotetext{
${ }^{4}$ Even though the subjects participated in groups of six, the experimenter largely controlled interactions and most dependent measures were individual responses. Thus, it can be argued that individual and not the group is the proper unit of analysis. Furthermore, preliminary analysis showed the subjects within groups effect to be nonsignificant at $p<.20$, therefore, this term was pooled with the error term and the individual was used as the unit of analysis.
} 


\begin{tabular}{lcccc}
\hline & $\underline{F}(1,88)$ & & Interpersonal Distance \\
Measure & & & Close & $\frac{F a r}{1}$ \\
How crowded were you? & 47.88 & & 5.7 & 1.9 \\
How confined were you? & 3.95 & 4.9 & 3.7 \\
Uncomfortable-comfortable & 12.19 & 4.2 & 3.1 \\
Confining-not confining & 20.95 & 4.6 & 2.8 \\
Restrictive-unrestrictive & 4.94 & 4.7 & 3.8 \\
Poorly arranged-wel1 arranged & 6.68 & 2.7 & 2.0 \\
Crowded-not crowded & 26.27 & 4.0 & 2.1 \\
\hline
\end{tabular}

Note. Al1 F-ratios were significant at $\underline{p}<.05$. Higher (more positive) means indicate greater perceived crowding.

was greater in crowded subjects if subjects had been warned that the drug would probably produce side effects than if they had been told it would have no noticeable effect, simple $F(1,88)=$ 9.50, $p<.05$.

A nearly identical interaction was obtained on subjects' ratings of their own reactions to the medication as measured by the "Symptom Checklist." Analysis of the average of the nine symptoms relevant to the described side effects information showed a main effect of the side effects, $F(1,88)=4.95, p<.05$, qualified by an interaction of side effects information and distance. Table 2 indicates that the difference due to side effects information was significant only when subjects were seated close together. Again, subjects in the close/side effects condition reported that they experienced the relevant symptoms to a greater degree than did subjects in the close/no side effects condition. Also, a distance $X$ sex interaction, $F(1,88)=4.72, p<.05$, indicated that the close females reported significantly fewer symptoms $(M$ $=1.2$ ) than both close males and far females (both $\mathrm{Ms}=1.5, p$ 


\begin{tabular}{|c|c|c|c|c|}
\hline \multirow[b]{3}{*}{ Measure } & \multicolumn{4}{|c|}{ Condition Means } \\
\hline & \multicolumn{2}{|c|}{ Side effects } & \multirow{2}{*}{$\begin{array}{l}\text { No Side } \\
\text { Close }\end{array}$} & \multirow{2}{*}{$\begin{array}{r}\text { effects } \\
\text { Far }\end{array}$} \\
\hline & Close & $\underline{\text { Far }}$ & & \\
\hline Self-reported Stress & 3.90 & 3.60 & 2.10 & 3.30 \\
\hline Symptoms Ratings & 1.58 & 1.48 & 1.20 & 1.45 \\
\hline Systolic Blood Pressure & -1.50 & $-5 \cdot 30$ & -8.60 & -3.60 \\
\hline Fear Factor Scores & -.12 & .32 & .01 & -.22 \\
\hline Friendliness of Others & 6.50 & 5.90 & 6.50 & 8.10 \\
\hline
\end{tabular}

Note. More positive means indicate greater stress, less decrease in blood pressure, greater fear and greater friendliness.

$<.05)$. The mean for far males was intermediate and did not differ from the other conditions $(M=1.4)$.

Finally, the distance $X$ information effect was obtained on physiological indices of stress. Records of systolic and diastolic blood pressure, pulse, and respiratory rate were taken both when subjects entered the experimental setting and again at the end of the task session. Change scores were computed for each measure by subtracting a subject's initial measure from the final measure; thus, a positive score indicates acceleration of a function, while negative scores indicate deceleration. Significant side effects information $X$ distance and side effects information $X$ sex interactions were obtained multivariately, $F_{s}(4,85)=2.47$ and 2.99 , respectively, both $p s<.05$. The side effects information $X$ distance interaction was significant univariately for change in systolic blood pressure. The pattern of means presented in Table 2 parallels that found for subjects' self-reported symptoms - systolic blood pressure was higher when close subjects expected the medication to have side effects than when they did not expect any side effects. The interaction between sex and side effects was signi- 
JOURNAL OF NONVERBAL BEHAVIOR

ficant univariately for changes in diastolic pressure, $F(1,88)=$ $6.24, p<.05$. The diastolic pressure of males who anticipated side effects of the drug was higher $(M=6.0, p<.05)$ than the diastolic pressure in males who did not expect any side effects $(M=11.7)$ and females who did $(M=-1.3)$. The mean diastolic pressure of females who did not expect side effects was intermediate $(M=$ 1.2).

The differences obtained in self-reported stress, symptoms, and systolic blood pressure strongly support the prediction that stress would be produced by close physical proximity only if accompanied by information that leads a negative appraisal of the situation. However, the strength of this effect was due not only to the high levels of stress displayed by subjects in the close/side effects condition, but also by the relatively low levels of stress experienced by persons in the close/no side effects condition. Table 2 shows that subjective stress, self-reported symptoms, and systolic blood pressure changes were significantly reduced in the close/no side effects condition relative to the far/no side effects condition; the simple $F_{s}(1,88)$ for this comparison were 4.05, 3.93, and 4.08 , respectively (all ps <.05). Thus, somewhat unexpectedly, when people were told that the drug they had taken would have no side effects and hence would only have beneficial consequences, sitting very close to others was less stressful than sitting father apart.

\section{Affective and Behavioral Effects of Crowding Stress}

Evaluations of self. Subjects' ratings of themselves on the 15 bi-polar adjectives were factor analyzed in order to group items into meaningful and empirically justifiable categories. A principle axes factoring with varimax rotations, using the within cells correlation matrix (computed by subtracting the appropriate cell mean from each subject's original score), yielded a three factor solution that accounted for $98.2 \%$ of the common variance. Items indicative of fear loaded highly on the first factor (e.g., afraid-unafraid, frightened-not frightened), while the remaining two factors were indicative of crowding (e.g., comfortable-uncomfortable, crowdednot crowded) and nervousness (e.g., nervous-not nervous, restlesscalm). These three factors accounted for approximately $75 \%$, $15 \%$, and $10 \%$ of the common variance, respectively.

Factor scores for these factors were computed and submitted to a muiltivariate analysis of variance, which revealed a main 
effect of distance and a marginally significant interaction of distance and side effects, $F(3,86)=4.05$ and 2.26 , ps $<.05$ and .09 , respectively. The main effect was significant only on the crowding factor, $F(1,88)=11.18, p<.05$. Subjects seated close together rated themselves as more crowded than subjects seated farther apart; the standardized factor score means were .21 and -.21 , respectively. The distance $X$ side effects interaction was significant univariately for self-ratings of fear, $F(1,88)=4.16, p<.05$. The means presented in Table 2 indicate that subjects seated far apart from one another felt more fearful when they expected that the drug would, rather than would not, have side effects $(p<.05)$. The means for the two close conditions fell intermediate and did not differ from any of the other conditions.

Perception of other group members. A multivariate analysis of variance of five items which assessed subjects' ratings of how pleasant they considered the group experience to be, how much they liked the group members, and the friendliness, nervousness, and aggressiveness of others revealed a main effect of subject sex and an interaction between side effects and interpersonal distance, $F s(5,84)=2.77$ and 2.70 , respectively, $p s<.05$. Males, as compared to females, liked other group members less $(\mathrm{Ms}=6.9$ and 7.8) and felt that others were less nervous ( $M s=3.7$ and 4.6). The side effects $X$ distance interaction was significant univariately for ratings of the friendliness of other group members. Table 2 indicates that subjects in the far/no side effects condition rated the others as more friendly than did subjects in all other conditions ( $p$ s $<.05)$.

Task performance. A multivariate analysis of performance on the anagram, memory, creativity, and judgments of Johnny Rocco tasks yielded only a sex main effect, $F(4,85)=3.24, p$ $<.05$. The effect was obtained univariately on the anagrams score, $F(1,88)=11.41, p<.05$, with females performing better than males. Females correctly completed a mean of 5.2 anagrams while males correctly solved 3.5. Worchel and Teddlie (1976) previously reported finding that subjective crowding increased punitiveness toward another. Using the same measure, however, the present study found no differences due to crowding. Indeed, the correlation between punitiveness and stress was only +.12 ( $n s$ ) and the correlation between ratings of crowding and punitiveness was -.01 (ns). 


\section{Causal Attributions}

The seven items requesting subjects to evaluate the causes of their behavior were divided conceptually into three groups. Three MANOVAs tested the effects of the three factors-side effects, interpersonal distance, and sex of subject-on subject's attributions. One MANOVA tested subjects' attributions about the influence of the medication on their feelings and task performance, one tested attributions to others as a cause of feelings and the third considered subjects' attributions to extraneous causes (distractions, being in an experiment, other) of their behavior. Multivariately, a significant effect of side effects, $F(2,87)$ $=5.41, p<.05$, was obtained on subjects' attributions to others as an influence. This effect was found univariately on both items, the influence of others on feelings, $F(1,88)=4.54, p<.05$ and task performance, $F(1,88)=5.46, p<.05$. The subjects who were expecting side effects thought that others were significantly more influential on both their feelings $(M=4.4)$ and task performance $(M=7.4)$ than did subjects not expecting side effects $(M s=3.3$ and 6.6 , respectively). Similarly, a multivariate effect was obtained on subjects' attributions to the medication, $F(4,87)=4.82, p<.05$. Subjects expecting side effects believed that the medication influenced their feelings ( $M=2.5$ ) but not their task performance more than subjects not expecting side effects $(M=2.3)$. A marginally significant interaction between sex of subject and the expectation of side effects was found multivariately, $F(2,87)=2.82, p$ $<.06$. All subjects expecting side effects attributed to the medication a large influence on their feelings (as would be expected from the information), but females who did not expect side effects also believed that the drug had a large influence on their feelings. Only male subjects who did not expect side effects reported that the drug had little influence on their feelings.

\section{DISCUSSION}

The results of this study indicate that close interpersonal proximity has differential effects on cognitions, task performance, and physiological arousal. Furthermore, the effects of a second source of stress combined with close interpersonal distance to have different effects on the various types of response.

Persons in the close interpersonal density conditions perceived themselves to be more crowded, confined, and uncom- 
fortable than those seated farther apart. Similarly, they rated the experimental setting as more crowded. No interactions with stress information were found on any self-reported subjective evaluations of crowding to the side effects of the "drug." Nor did they feel they were more "crowded" than subjects in the close/no side effects condition, even though the close/side effects subjects were actually subjectively and physiologically more aroused. When asked to rate how crowded they felt, these subjects appeared to consider not their internal state of arousal but the actual density conditions to which they were exposed. Subjects' self-reports of feeling crowded appeared to be an objective cognitive assessment of spatial conditions.

The lack of a misattributional effect contrasts with findings reported by Worchel and Teddlie (1976) and Worchel and Yohai (1979). Both of the groups claimed to have prevented a crowding attribution by distracting subjects from the proximity of others. However, as they did not assess subjects' levels of arousal it is unclear whether the distractions interfered with the crowding attribution or reduced the arousal of the subjects. Worchel and Teddlie believed that their close subjects were aroused and misattributed the arousal but reduced arousal would have eliminated the need for a causal attribution at all. This may account for the discrepancy between the present results and their reported results. However, Worchel and Yohai used a concurrent stressor that should have increased arousal and they did find a misattribution effect.

Psychophysiological measures and subjective reports of stress indicated that subjects felt most stressed when they were crowded and were expecting stressful side effects. Subjects felt least stressed when they were crowded and expecting no side effects. This pattern of results is not what would be expected if the effects of the stressors combined additively. An additive model would predict most stress when both stressors are present, intermediate levels of stress when one stressor is present alone and lowest levels of stress when neither stressor is present.

In the present study crowded individuals not anticipating side effects felt less stressed than subjects who were neither crowded nor expecting a stressful event. Table 2 shows that the crowded/no side effects subjects had the lowest levels of self-reported stress, lowest number of symptoms reported, and lowest systolic blood pressure of all subjects. The pattern of results of self-reported and psychophysiological stress raises the question of whether crowding 
in itself is necessarily arousing or aversive. Rather, the crowded condition appeared to facilitate stress-reduction under certain circumstances.

Factor analyses of subjective feelings suggested that the feelings we assessed fell into three groups. One group of items dealt with feelings of crowding and these increased in dense conditions. A second group dealt with feelings of fearfulness. Subjects' feelings of fear were sensitive to both density and side effects information. When subjects expected to experience side effects, sitting far apart increased their fear, but when subjects expecting side effects sat very close together they felt less afraid. When subjects did not anticipate side effects, interpersonal distance had no effect on fear. This pattern of results is consistent with the social comparison fear reduction hypothesis (Schachter, 1959). Subjects in the side effects conditions were afraid of possible, probably somewhat unknown and mysterious, negative side effects that they might experience. The close proximity to others may have promoted access to social comparison information, facilitating fear reduction in the high density condition, while the distant spacing of others in the far condition may have inhibited fear reduction due to social comparison. Subjects in the no side effects condition expected no such fearful experience and social comparison was not relevant to them.

These results suggest that crowding may act as either a positive or negative influence depending upon the nature of other stressful stimuli in the environment. At times crowding may facilitate fear reduction through social comparison, sharing a common destiny with others, or other mechanisms. In these situations we might expect proximity to be comforting and pleasant. In other situations we may find ourselves trying to cope with other stressful stimuli that are exacerbated by crowding and the crowded conditions only add to the stress experienced.

The pattern of results on self-reported and psychophysiological stress effects is reminiscent of Freedman's (1975) density-intensity hypothesis. Freedman proposed that crowding (density) by itself has neither good nor bad effects but acts to intensify the individual's reactions to the situation. While the present authors do not propose this explanation to explain all effects or to operate across all situations, in this experiment high density appeared to intensify stressful effects when they were present and, likewise, the presence of others in relatively unstressful circumstances appeared to be stress-reducing. 
TEDRA A. WALDEN, DONELSON R. FORSYTH

Subjects' causal attributions did not parallel either the stressors to which they were exposed or their own pattern of responses. Subjects who reported feeling crowded did not attribute causal influence to the crowded conditions. Subjects expecting side effects, however, believed that both the drug and the close presence of others affected their feelings and task performance. Task performance was not different in the side effects conditions, however. Nor were the "feelings" (stress, symptoms, psychophysiological responses) that subjects experienced objectively attributable solely to the side effects manipulation. Thus, subjects who believed that they would be experiencing arousal from a particular source did attribute causal influence to that source, even though the pattern of stress experience was not as simple as that. In this study the expectation of side effects may have been so salient to causal attributions that other potential sources of arousal (crowding) were swamped.

A sex difference in self-reported symptoms indicated that physically close females reported fewer symptoms than did close males. This is consistent with prior research (Freedman, Levy, Buchanan, \& Price, 1972; Stokols, Rall, Pinner, \& Schopler, 1973). One explanation for this difference suggests that females are partially insulated from the noxious effects of crowding because of their focus on interpersonal relations with others. Epstein and Karlin (1975) found that females displayed greater cohesiveness and cooperation than males in a high density situation. They suggested that the capacity of a cohesive group to share distress may enhance adjustment to the discomforts of excessive interpersonal proximity. Thus, for females, liking others provides a stronger barrier against crowding-induced stress than it does for males. Supporting this speculation, the correlation between the rated friendliness of others and stress was +.15 for males, but -.38 for females. While the correlation for males is not significantly different from zero, that for females is $(p<.05)$, and the correlations are significantly different from one another $(p<.05)$. For females, being in a friendly group may help reduce the level of stress.

Crowding and situational information were not found to influence task performance. Since the tasks included were considered to be fairly complex (memory, creativity, anagram solving), we expected task decrements in the crowded conditions. It may have been the case, though, that to this well-educated sample of subjects the tasks were not complex enough to show decrements. It is also possible that because all but one task required individual 
rather than group responses and did not depend on cooperation of others, crowding did not inhibit goal completion and hence was not detrimental (Heller, Groff, \& Solomon, 1977; Schopler \& Stockdale, 1977). Regardless, it is important to note that performance on these tasks was not as sensitive an indicator of crowding or general stress as were cognitive and physiological indices.

The present study found that excessive interpersonal proximity has different effects on subjective feelings, cognitions, task performance, and psychophysiological responses. This highlights the importance of including assessments of multiple types of responses in investigations of crowding on humans. In this study reports of subjective crowding did not necessarily parallel effects on psychophysiological symptoms of stress or task performance. Self-reports of stress did parallel psychophysiological measures related to stress, although the latter showed stronger effects. Nisbett and Wilson (1977) have argued that people often cannot report accurately on the effects of particular stimuli on their behavior, indeed, that the introspective process is so poor that the reliability and accuracy of subjective reports must often be seriously questioned. Our results show that, while subjective reports may parallel more objective assessments, this is not necessarily the case, especially when generalizing across different types of responses.

One striking feature of this study is the lack of main effects of crowding unqualified by interactions with other factors. Only the subjects' self-reports of feeling crowded were an unqualified main effect of density conditions. Other responses - physiological, selfreports of stress, fear, and attributions as to the causes of feelings and performance - interacted with other factors. The lack of consistent, clear-cut main effects of crowding is not unique to this study. Rather, others have been prompted by this pattern of results to hypothesize that additiona! causal variables such as goal interference (Schopler \& Stockdale, 1977) physical interaction (Heller et al., 1977) or lack of privacy (Walden, Nelson, \& Smith, 1981) may be necessary for negative effects of crowding to surface. The present study adds to these the qualification that under some circumstances extremely close proximity may actually be stress-reducing. This stress-reduction effect occurred even though subjects considered themselves to be crowded. These findings seriously question the conceptualization of density or crowding as necessarily stressful, perhaps suggesting that crowding may be a 
mediating variable, exerting its effects primarily through its interaction with other aspects of the situation.

The present study raises several questions for further research. It will be important to predict which responses will be influenced in situations in which a variety of factors, positive and negative, combine to influence behavior. If high density does sometimes act to intensify positive and negative reactions to a total situation, what is the "neutral" line that distinguishes responses which will become more negative from those which become more positive? What is the effect of high density on an otherwise neutral situation? Finally, it will be important to investigate further, as did Worchel and Teddlie (1976) and Langer and Saegert (1977) the causal influence of cognitions upon other responses to crowded situations.

\section{Reference Notes}

1. Schopler, J., McCallum, R., \& Rusbult, C. Behavioral interference and locus of control as determinants of being crowded. In preparation, University of North Carolina at Chapel Hill, 1978.

2. Epstein, Y, \& Aiello, 1. Effects of crowding on electrodermal activity. Paper presented at the American Psychological Association, New Orleans, 1974.

3. Heshka, S., \& Pylypuk, A. Human crowding and adrenocortical activity. Paper presented at the Canadian Psychological Association, Quebec, 1975.

4. Barefoot, J., \& Kleck, R. The effects of race and physical proximity of a co-actor on the social facilitation of dominant responses. Unpublished manuscript, Carleton University, 1970.

\section{REFERENCES}

Aiello, J., Epstein, Y., \& Karlin, R. Effects of crowding on electrodermal activity. Sociological Symposium, Spring, 1975.

Aiello, J., Nicosia, G., \& Thompson, D. Physiological, social, and behavioral consequences of crowding on children and adolescents. Child Development, 1979, 50, 195-202.

Calhoun, J. Population density and social pathology. Scientific American, 1962, 206, 139-148.

Christian, J. Phenomena associated with population density. Proceedings of the National Academy of Science, 1961, 47, 428-449.

Dabbs, I.M. Physical closeness and negative feelings. Psychonomic Science, 1971, 23, 141-143.

D'Atri, D. Psychophysiological responses to crowding. Environment and Behavior. 1975, 7, 237-252.

Efran, M.G., \& Cheyne, J.A. Affective concomitants of the invasion of shared space: Behavioral, physiological, and verbal indicators. Journal of Personality and Social Psychology, 1974, 29, 219-226.

Epstein, Y., \& Karlin, R.A. Effects of acute experimental crowding. Journal of Applied Social Psychology, 1975, 5, 34-53. 
Evans, G.W. Behavioral and physiological consequences of crowding in humans. Journal of Applied Social Psychology, 1979, 9, 27-46.

Evans, G.W., \& Howard, R.B. Personal space. Psychological Bulletin, 1973, 80, 334-344.

Feather, N.T. Attribution of responsibility and valence of success and failure in relation to initial confidence and task performance. Journal of Personality and Social Psychology, 1967, 6, 129-144.

Freedman, J. Crowding and behavior. San Francisco: Freeman, 1975.

Freedman, J., Heshka, S., \& Levy, A. Population density and pathology: Is there a relationship? Journal of Experimental Social Psychology, 1975, 11, 539-552.

Freedman, J., Klevansky, S., \& Erlich, P. The effect of crowding on human task performance. Journal of Applied Social Psychology, 1971, 1, 7-25.

Freedman, J.L., Levy, A.S., Buchanan, R.W., \& Price, J. Crowding and human aggressiveness. Journal of Experimental Social Psychology, 1972, 8, 528-548.

Galle, O., Gove, W., \& McPherson, I. Population density and pathology: What are the relations for man? Science, 1972, 176, 23-30.

Griffitt, W., \& Veitch, R. Hot and crowded: Influences of population density and temperature on interpersonal affective behavior. Journal of Personality and Social Psychology, 1971, 17(1), 92-98.

Heller, J.F., Groff, B.D., \& Solomon, S.H. Toward an understanding of crowding: The role of physical interaction. Journal of Personality and Social Psychology, 1977, 35(3), 183-190

Langer, E., \& Saegert, S. Crowding and cognitive control. Journal of Personality and Social Psychology, 1977, 35(3), 175-182.

Loo, C. Important issues in researching the effects of crowding on humans. Representative Research in Social Psychology, 1973, 4, 219-226.

Lywood, P. Blood pressure. In P. Venables \& S. Martin (Eds.), A manual of psychophysiological methods. Amsterdam: North-Holland, 1967.

McBride, G., King, M. \& James, J. Social proximity effects on galvanic skin responses of adult humans. Journal of Psychology, 1965, 61, 153-157.

McCallum, R., Rusbult, C., Hong, G., Walden, T., \& Schopler, J. Effects of resource availability and the importance of behavior upon the experience of crowding. Journal of Personality and Social Psychology, 1979, 37, 1104-1113.

Nisbett, R.E., \& Wilson, T.D. Telling more than we can know: Verbal reports on mental processes. Psychological Review, 1977, 84, 231-259

Schachter, S. The psychology of affiliation. Stanford, California: Stanford University Press, 1959.

Schopler, J., \& Stockdale, J. An interference analysis of crowding. Environmental Psychology and Nonverbal Behavior, 1977, 1, 81-88.

Sequin, C. The individual space. International Journal of Neuropsychiatry, 1967, 3, 108-117.

Sherrod, D. Crowding, perceived control and behavioral aftereffects. Journal of Applied Psychology, 1974, 4, 171-186.

Stokols, D. On the distinction between density and crowding: Some implications for future research. Psychological Review, 1972, 79, 275-277.

Stokols, D., Rall, M., Pinner, B., \& Schopler, J. Physical, social, and personal determinants of the perception of crowding. Environment and Behavior; 1973, 5(1), 87-115.

Sundstrom, E. An experimental study of crowding: Effects of room size, intrusion, and goal blocking on nonverbal behavior, self-disclosure, and self-reported stress. Journal of Personality and Social Psychology, 1975, 32, 645-654.

Walden, T., Nelson, P., and Smith, D.E. Crowding, privacy, and coping. Environment and Behavior, 1981, 13, 205-224.

Worchel, S. and Teddlie, C. The experience of crowding: A two-factor theory. Journal of Personality and Social Psychology, 1976, 34, 30-40.

Worchel, S. and Yohai, S.M. The role of attribution in the experience of crowding. Journal of Experimental Social Psychology, 1979, 15, 91-104. 